\title{
WDM-PON Delivering 5-Gbps Downstream/2.5-Gbps Upstream Data
}

\author{
Balaji Raobawale \\ P. G. Department \\ M.B.E.S. College of Engineering, Ambajogai, India
}

\author{
S. K. Sudhansu \\ P. G. Department \\ M.B.E.S. College of Engineering, Ambajogai, India
}

\begin{abstract}
We experimentally demonstrate wavelength division multiplexing passive optical network system delivering downstream 5 -Gbps data and upstream 2.5 -Gbps data over $153 \mathrm{~km}$ single mode fiber on a single wavelength using pulse source that is mode-locked laser generating a single pulse of "sech" shape with specified power and width, that is, soliton pulse. The optical source for downstream data and upstream data is soliton pulse generator at central office and reflective semiconductor optical amplifier at each optical network unit. We also investigate analysis of backscattered optical signal for upstream data and downstream data simultaneously. Bit error rate and Quality factor was measured to demonstrate the proposed scheme.
\end{abstract}

\section{Keywords}

Wavelength division multiplexing passive optical network, soliton pulse, reflective semiconductor optical amplifier, erbium doped fiber amplifier, single mode fiber, photo detector, arrayed waveguide grating.

\section{INTRODUCTION}

To meet ever-increasing demands for higher speed and advanced services in access networks require a bandwidth of above $50 \mathrm{Mbps}$ for next generation services to end users [1]. The use of technologies based on optical fibers can easily achieve bandwidths higher than 100Mbps and at the same time can reduce maintenance and repair costs $[1,2]$. In terms of cost, a passive optical network (PON) is very attractive, because there are no active components in the transmission line. A PON system typically consists of an optical line terminal in a central office (CO), a remote node (RN), and optical network units (ONUs). There are limitations on the transmission capacity and number of users of time-division multiplexing (TDM) PONs with splitters, but they are easy to install, are small, and require no electricity [3]. On the other hand, a wavelength division multiplexing (WDM) PON with arrayed waveguide gratings (AWGs) assigns a different wavelength channel to each end user, so the bandwidth can be high. In addition, it is far superior to TDM-PONs in security $[3,4]$ and potentially cost effective [5]. The development of colorless ONUs is a key issue in WDM-PON technologies to reduce the system cost dramatically. Among various solutions, the use of a reflective semiconductor optical amplifier (RSOA) in an ONU is a good candidate, because this approach has the flexibility to assign a wavelength to the upstream signal, and the signal is directly modulated without an external modulator and amplified at the same time [6]. A hybrid WDM/TDM-PON system that combines the TDM and WDM-PON systems was recently developed [7]. The hybrid system combines the high bandwidth capacity of WDM-PON and the bandwidth efficiency of TDM-PON, thereby achieves the accommodation of great number of ONUs, while the high bandwidth per ONU is maintained. On the other hand, there has recently been a strong demand for so called "triple play" broadband access services including internet, internet protocol telephony and video broadcasting. A video overlay technique has been also proposed in a WDM-PON system using an MIFPLD as a BLS for broadcasting and upstream transmission [8]. Unlike other video overlay techniques, this does not require the modification of either $\mathrm{RN}$ at outdoor or the transmitters at $\mathrm{CO}$ to deliver the broadcast signals to ONU. However, this technique should use an external modulator devoted to the broadcast signals. In addition, as this technique assigned a dedicated wavelength band to the broadcast signals, the ONU should be able to accommodate three wavelengths for the broadcasting and up/downstream baseband data services in the worst case. These, ultimately, increase the system cost and make worse bandwidth utilization, which weaken the original purpose of developing a low-cost. In this article, we propose and demonstrate WDMPON system delivering downstream data and upstream data on a single wavelength using pulse source that is mode-locked laser generating a single pulse of "sech" shape with specified power and width, that is, soliton pulse. To improve wavelength utilization of network, we adopt the transmission of downstream and upstream data on a single wavelength. This not only reduces the required number of optical sources and wavelength but also relieves complexity in WDM-PON. We also demonstrate the propagation of soliton pulse in optical fiber. The existence of solitons in optical fiber is the result of a balance between the chirps induced by fiber dispersion characterized by group velocity dispersion coefficoeffi $\beta_{2}$ and fiber nonlinearity characterized by self phase modulation coefficient $\gamma[9,10]$. Today's optical fiber system is limited by linear and nonlinear effect. We demonstrate the propagation of soliton pulse in optical fiber, so that our scheme will be a practical solution to meet the data rate and cost-efficient of the optical links simultaneously in tomorrow's WDM-PON access networks. Performance of proposed network is evaluated by transmitting downstream 5Gbps data over $153 \mathrm{~km}$ single mode fiber (SMF) and upstream 2.5 -Gbps data over $153 \mathrm{~km} \mathrm{SMF}$. We also investigate analysis of backscattered optical signal for upstream and downstream data simultaneously. We present single wavelength transmitssion in this article for sake of simplicity.

\section{OPERATIONAL PRINCIPLE}

Figure 1 show operation principles of proposed system, which consists of a pseudorandom sequence generator. A pulse source is mode-locked laser that is generating a single 


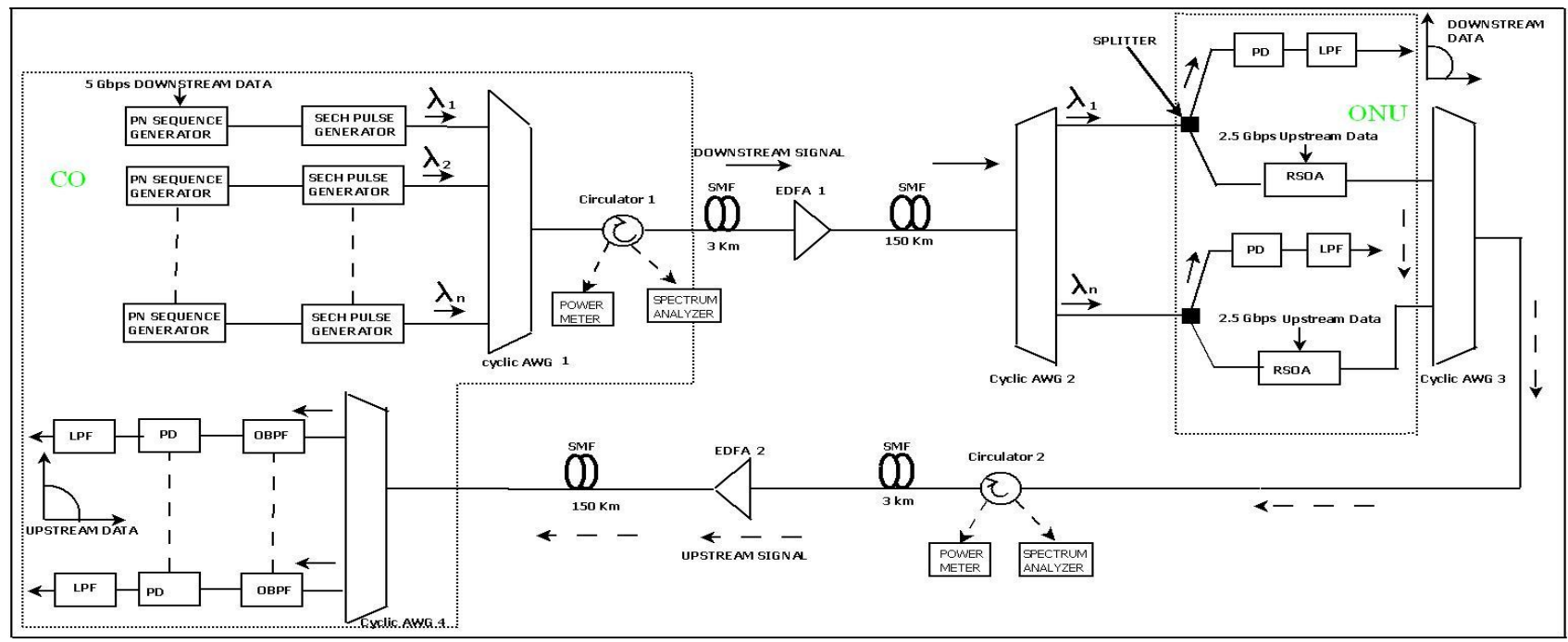

Figure 1. The schematic diagram of the proposed WDM-PON

pulse of sech shape with specified power and width, that is, soliton pulse. Here, sech pulse generator is nothing but modelocked laser which generate soliton pulse. It is possible to generate soliton pulse for different wavelengths with specified peak power level. The downstream data is transmitted over soliton pulse, and the output of sech pulse generator is given to array waveguide grating 1 (AWG 1), which is used for multiplexing of downstream signal. The modulated downstream signals are wavelength multiplexed using AWG 1, transmitted through circulator 1 to analyze backscattered signal. All these downstream signal are transmitted via single mode fiber (SMF) which is practically used. To strengthen signal erbium doped fiber amplifier (EDFA) is used as preamplifier. At the end downstream link wavelength demultiplexed by AWG 2 and send to corresponding ONU. At circulator 1 backscattered optical signal for downstream data signal can be measured using optical power meter and spectrum analyzer. In ONU, optical signal is splitted by splitter, half of optical signal is detected by photo detector (PD) for reception of downstream data signal and output of PD given to low pass filter. The other half of optical signal is injected by RSOA for remodulation of RSOA with the upstream baseband data. The RSOA is operating at the gain saturation region can squeeze out downstream baseband data and enable the upstream data to be imposed directly upon downstream signal directly [11]. The remodulated upstream signals are wavelength multiplexed by AWG 3, transmitted back to $\mathrm{CO}$ via circulator 2, different fiber, EDFA and then demultiplexed by AWG 4. At circulator 2, backscattered optical signal for upstream data signal can be measured using optical power meter and spectrum analyzer[12]. At CO, upstream data signal is detected by PD for reception of upstream data signal and output of PD given to low pass filter.

\section{SIMULATION SET-UP}

Figure 2 shows simulation set-up for proposed scheme. We present single wavelength $1550 \mathrm{~nm}$ transmission in this article for sake of simplicity. Soliton was modulated at downstream data $5 \mathrm{Gbps}$ with pseudorandom bit sequence with length of $2^{\mathrm{aI}}-1$. At $5 \mathrm{Gbps}$, initial pulse width is $14.18 \mathrm{ps}$. The peak power necessary to launch a fundamental soliton pulse can be calculated by

$$
\mathbb{R}_{\mathbb{N}}=\frac{\mathbb{N}^{\mathbb{2}}\left\|\beta_{\mathbb{N}}\right\|}{7 T_{\mathscr{Q}}^{\mathbb{2}}}
$$

where $\mathbb{R}_{\mathbb{N}}$ - peak power necessary to launch a fundamental soliton pulse; $\mathrm{N}$-order of soliton pulse;

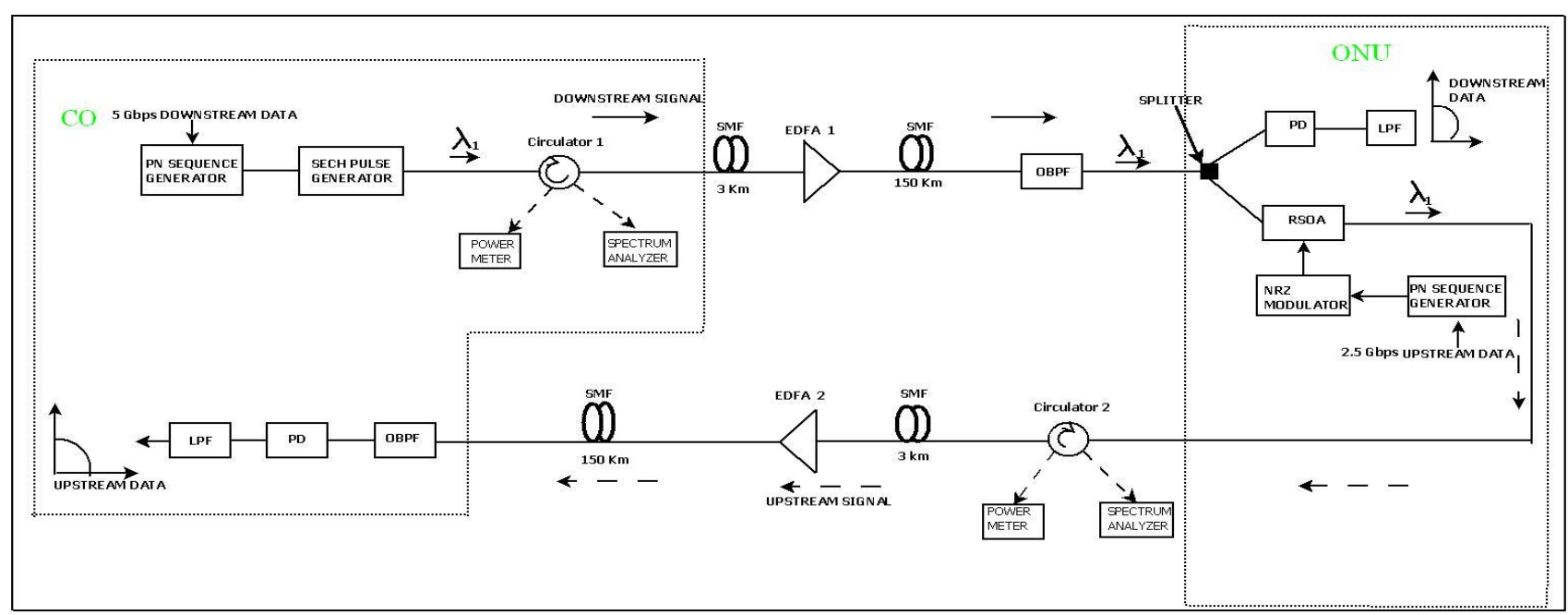

Figure 2. Simulation set-up for proposed scheme. 
$\beta_{2}$-group velocity dispersion coefficient, that is, fiber dispersion parameter; $\mathbb{T}_{\mathscr{0}}$-initial pulse width; $\gamma$-self phase modulation coefficient, that is, nonlinear parameter. For given $\mathrm{N}=1$ for fundamental soliton, , $\mathrm{n}_{2}=2.6 e^{-20} \mathrm{~m}^{2} / \mathrm{w}, \mathrm{A}_{\text {eff }}$ $=$ core effective area $=60 \mathrm{~lm}^{2} ; \mathrm{k}=1550 \mathrm{~nm}$ wavelength; $\mathrm{a}=$ attenuation $=0.2 \mathrm{~dB} / \mathrm{km} ; \beta_{2}=20 \mathrm{ps}^{2} / \mathrm{km} ; \mathbb{T}_{\mathbb{Q}}=14.18 \mathrm{ps}$.

Nonlinear parameter calculated as

$$
\gamma=2 \pi n_{2} / \lambda \mathrm{A}_{\text {eff }}
$$

For above given value finally got $\gamma=1.75 \mathrm{e}^{-11} \mathrm{~m}^{2} / \mathrm{w}$ and $\mathbb{P}_{\mathrm{N}}=7.56 \mathrm{dBm}$

Using above parameter, soliton pulse generated using modelocked laser at power $7.56 \mathrm{dBm}$ and $\lambda=1550 \mathrm{~nm}$ wavelength. Soliton was modulated at downstream data 5Gbps. Downstream data signal passes through single mode fiber of length $153 \mathrm{~km}$. Soliton can maintain their shape by amplifying fiber link, erbium doped fiber amplifier is used at distance $3 \mathrm{~km}$ from $\mathrm{CO}$ for downstream link. The Downstream signal is given to erbium doped fiber amplifier after $3 \mathrm{~km}$ to amplify the weak signal and then passed over $150 \mathrm{~km}$ fiber. At the end of link, optical band pass Bessel filter, which is having bandwidth $=4 *$ bit rate for downstream signal is used. The analysis of backscattered signal for downstream data signal is done at circulator 1 by calculating optical power of backscattered signal. Optical signal is splitted by splitter, half of optical signal is detected by PD for reception of downstream data signal and output of PD given to low pass Bessel filter which having cut off frequency $0.75 *$ bit rate to get better signal to noise ratio. The other half of optical signal is injected by RSOA for remodulation of RSOA with the upstream baseband data 2.5Gbps Table 1, simulation parameters for RSOA are shown. After remodulation of RSOA with the upstream baseband data $2.5 \mathrm{Gbps}$ upstream signal having optical power $-36.733 \mathrm{dBm}$ at output of RSOA. This upstream signal is passed via circulator 2, single mode fiber of total length $153 \mathrm{~km}$ with erbium doped fiber amplifier at $3 \mathrm{~km}$ from ONU on upstream link. The upstream signal is given to erbium doped fiber

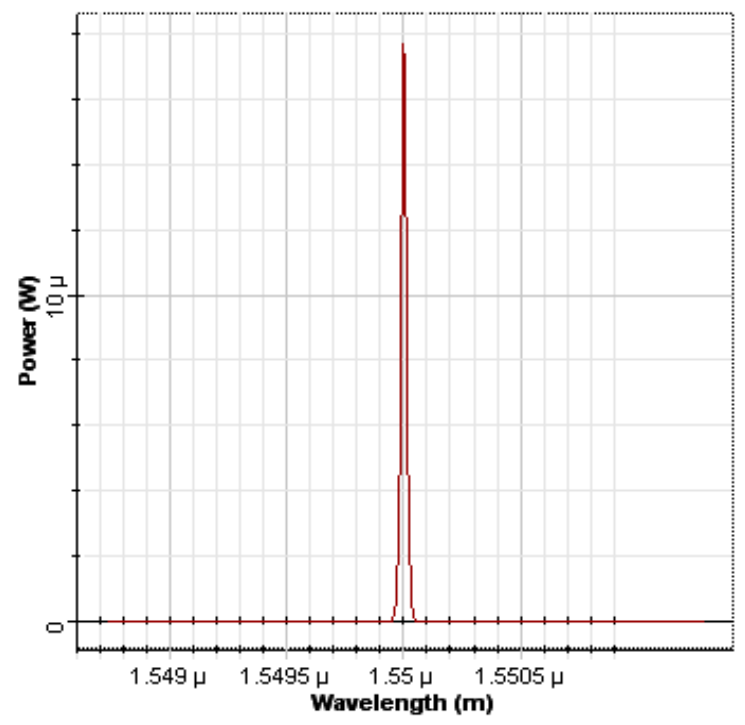

Table 1. Simulation Parameter for RSOA

\begin{tabular}{|l|c|}
\hline \multicolumn{2}{|c|}{ RSOA Parameters } \\
\hline Input facet reflectivity & $5 \mathrm{e}^{-5}$ \\
\hline Output facet reflectivity & $5 \mathrm{e}^{-5}$ \\
\hline Active length & $700 \mathrm{e}^{-6} \mathrm{~m}$ \\
\hline Taper length & $700 \mathrm{e}^{-6} \mathrm{~m}$ \\
\hline Width & $0.4 \mathrm{e}^{-6} \mathrm{~m}$ \\
\hline Height & $0.4 \mathrm{e}^{-6} \mathrm{~m}$ \\
\hline Optical confinement factor & 0.45 \\
\hline Nonlinear gain parameter & $1.2 \mathrm{e}^{-22} \mathrm{~m}^{2}$ \\
\hline
\end{tabular}

amplifier after $3 \mathrm{~km}$ to amplify the weak signal and passed over $150 \mathrm{~km}$ fiber. The analysis of backscattered signal for upstream data signal is done at circulator 2 by calculating optical power of backscattered signal. At the end of link, optical band pass Bessel filter is used which having the bandwidth $=4 *$ bit rate for upstream signal. Upstream optical signal is detected by PD for reception of upstream data signal and output of PD given to low pass Bessel filter which having the cut off frequency $0.75 *$ bit rate for upstream data to get better signal to noise ratio. Finally, BER performance of downstream data and upstream data signal was measured.

\section{RESULTS AND DISCUSSION}

For performance measurement, single channel was selected, the spectrum of that channel at the output of sech pulse generator as presented in Figure 3(a). Most of the optical power of that channel from sech pulse generator output

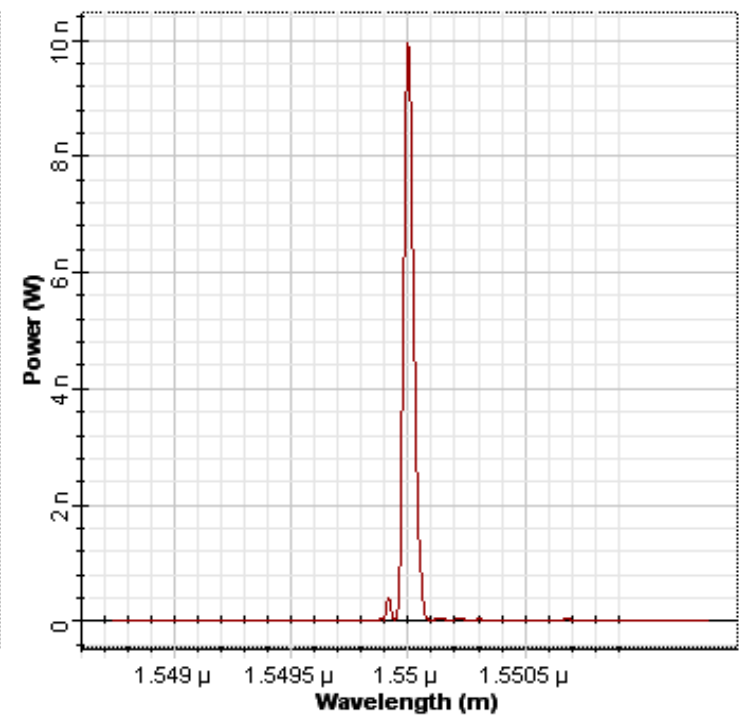

Figure 3 Optical spectrum (a) at output of sech pulse generator and (b) at output of RSOA. 


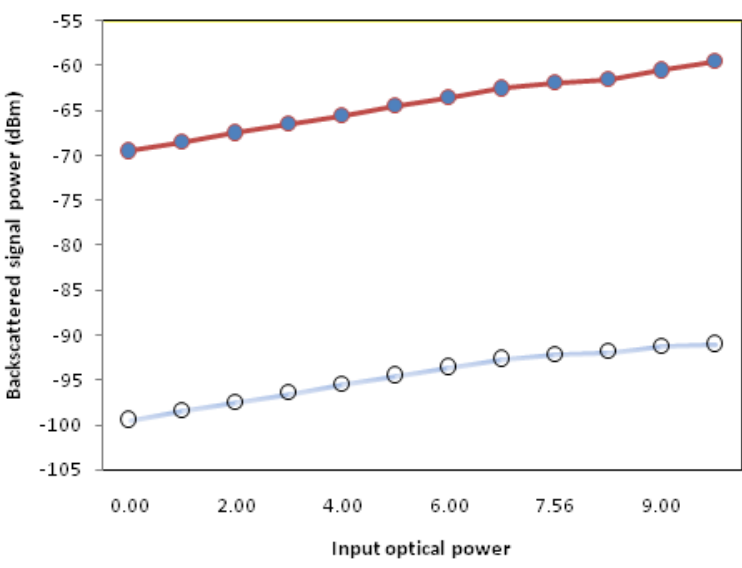

O- Input optical powervs backscattered power upstre am data - Input optical powervs backscattered power downstre am data

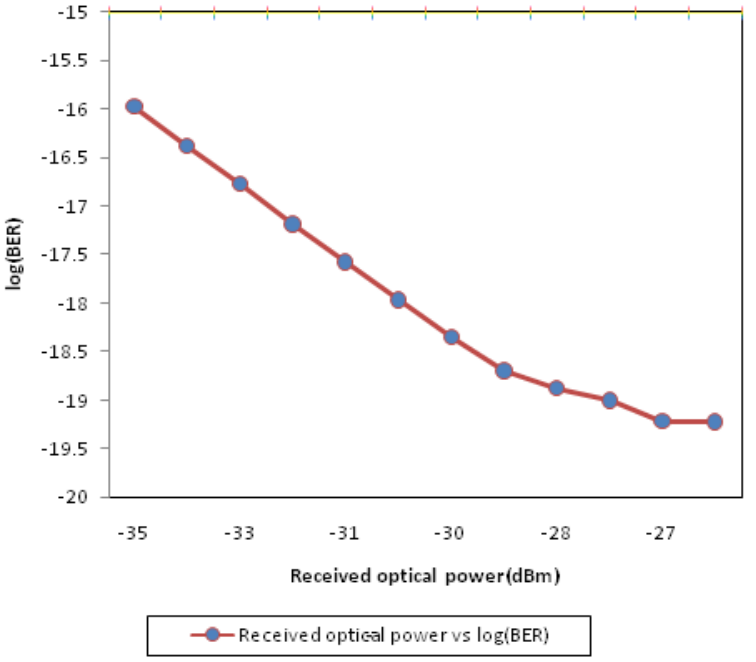

$\rightarrow$ Receive d optical power vs $\log (\mathrm{BER})$

Figure 4 (a) Detected total backscattered optical power and (b) transmission performance of downstream data
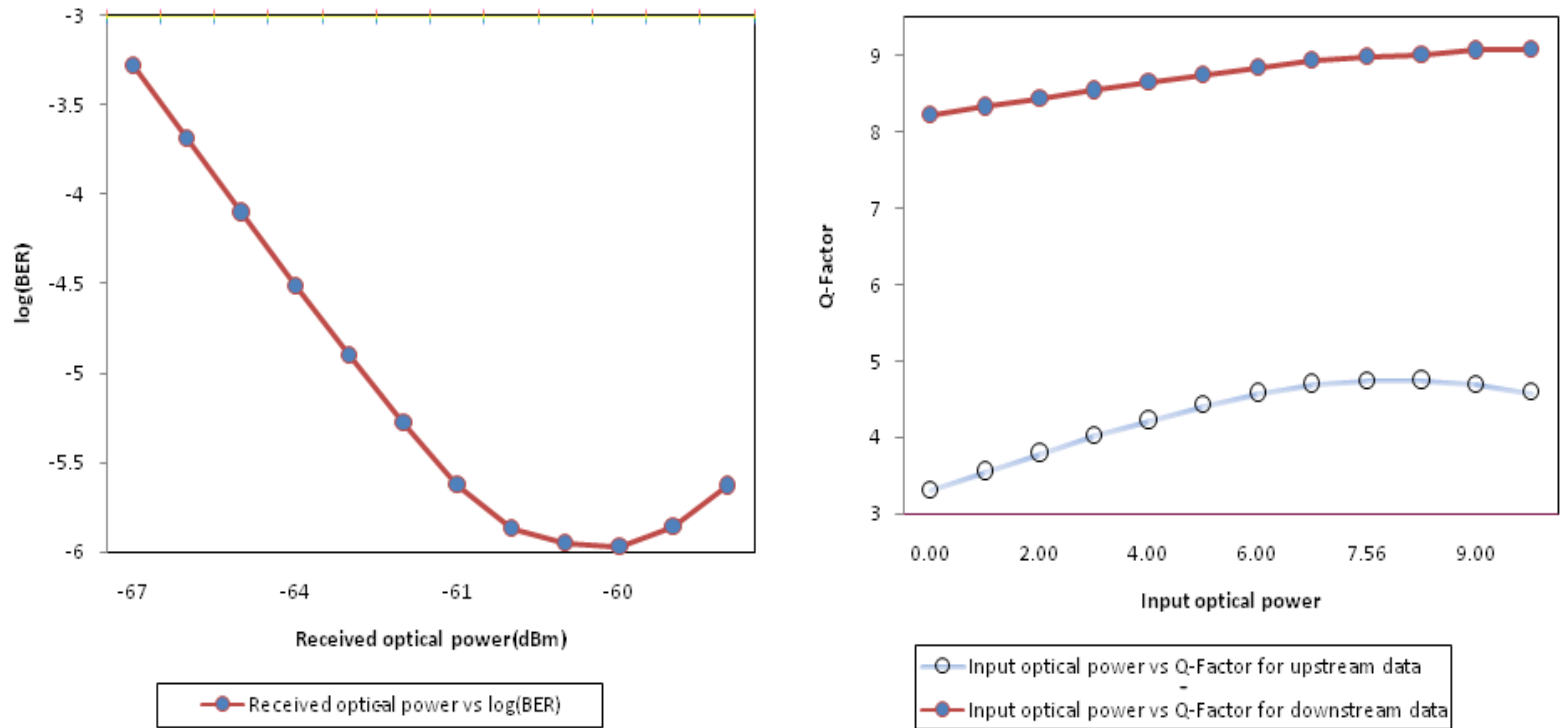

Figure 5 (a) Transmission performance of upstream data and (b) simulated Q-factor for downstream and upstream data.

was $-6.943 \mathrm{dBm}$ which was large, which did satisfy the requirement of injected optical power of RSOA to work under the gain saturation condition. In this condition, the output optical power of RSOA is about $-36.733 \mathrm{dBm}$ and spectrum at the output of RSOA as presented in Figure 3(b). First, we assessed Rayleigh scattering effect that reflects back to source. In this condition, the output optical power of RSOA is about $-36.733 \mathrm{dBm}$ and spectrum at the output of RSOA as presented in Figure 3(b). The scheme depicted in Figure 2 was utilized to study the influence of backscattered power in the downstream and upstream data transmission simultaneously. In Figure 4(a), detected total backscattered optical power in the downstream and upstream data transmission simultaneously is shown. As input optical power increases the backscattered signal power increases. In Figure 4(a), it is observed that the backscattered signal power is large in case of downstream data signal transmission as compare to upstream data signal transmission. There is too much reduction in backscattered signal power for our proposed scheme. In this article, only BER curve for both downstream and upstream data of channel $1550 \mathrm{~nm}$ were measured as shown in Figures 4(b) and 5(a) respectively. Obviously, the downstream and upstream performances increase with increase in received optical power. From these results, we compared the performance of downstream and upstream data Obviously, the performance of downstream is better than upstream. In Figure 5(b), simulated Q-factor for downstream and upstream data signal is shown. Obviously, the Q-factor for downstream and upstream data increases with increase in input optical power. From these results, we compared the performance of downstream and upstream data then obviously the performance of downstream is better than upstream data signal. From the above result, it is observed that BER for downstream data signal is same for input optical powers 7.56 and $7.5 \mathrm{dBm}$, and also it is true about Q-factor of downstream data signal. 


\section{CONCLUSIONS}

We experimentelly demonstrated WDM-PON system delivering downstream 5-Gbps data and upstream 2.5-Gbps data over $153 \mathrm{~km}$ SMF on a single wavelength using pulse source that is mode-locked laser generating a single pulse of sech shape with specified power and width, that is, soliton pulse. Since we have used single EDFA for each link so system will be cost-effective and also transmission distance of the proposed WDM-PON system can be expanded, while the performance is maintained. We have successfully demonstrated the propagation of soliton pulse in optical fiber, so our scheme will be a practical solution to meet the data rate and cost efficient of the optical link simultaneously in tomorrow's WDM-PON access networks and also solution for today's optical fiber system, which is limited by linear and nonlinear effect.

\section{REFERENCES}

[1] S.J. Park, C.H. Lee, K.T. Jeong, H.J. Park, J.G. Ahn, and K.H. Song, "Fiber to the home services based on wavelength division multiplexing passive optical network", J Lightw Technol 22 (2004), 2582-2591.

[2] H. Shinohara, "Broadband access in Japan: Rapidly growing FTTH market”, IEEE Commun Mag 43 (2005), $72-78$.

[3] J.J. Yoo, H.H. Yun, T.Y. Kim, K.B. Lee, M.Y. Park, B.W. Kim, and B.R. Kim, "A WDM-ethernet hybrid passive optical network architecture", In: Proc. ICACT, 2006, Vol.3, nos. 20-22, pp. 1754-1757.

[4] G. Maier, M. Martinelli, A. Pattavina, and E. Avadori, "Design and cost performance of the multistage WDM-
PON access networks", J Lightw Technol 18 (2000), $125-139$.

[5] C.H. Lee, W.V. Sorin, and B.Y. Kim, "Fiber to the home using a PON infrastructure", J Lightw Technol 24 (2006), 4568-4583.

[6] S.J. Park, G.Y. Kim, and T.S. Park, "WDM-PON system based on the laser light injected reflective semiconductor optical amplifier", Opt Fiber Technol 12 (2006), 162 169.

[7] F. Payoux, P. Chanclou, T. Soret, N. Genay, and R. Brenot, "Demonstration of a RSOA-based wavelength remodulation scheme in 1.25 git/s,bidirectional hybrid WDM-TDM PON", In: Proc. OFC, 2006, TuC4.

[8] J.-H. Moon, K.M. Choi, and C.H. Lee, "Overlay of broadcasting signal in a WDM-PON", In: Proceedings of the Optical Fiber Communication and Conference, 2006, Paper OThK8.

[9] G.P. Agrawal, "optical pulse propagation in doped fiber amplifier", Phys Rev A 44 (1991), 7493-7501.

[10] G.P. Agrawal, "Nonlinear fiber optics", 3rd ed., Academic Press, Orlando, FL, 2001.

[11] W.R. Lee, M.Y. Park, S.H. Cho, J. Lee, C. Kim, G. Jeong, and B.W. Kim, "Bidirectional WDM-PON based on gain-saturated reflective semiconductor optical amplifiers", IEEE Photon Technol Lett 17 (2005), 2460 2462.

[12] L Tawade and S Deosarkar "cost-effective wavelength division multiplexing passive optical Network delivering upstream/downstream data on a single wavelength using soliton pulse", microwave and optical technology letters, vol.54,No.2,February2012. 